Cahiers de la recherche sur les droits

Cahiers

Fon les Droits fondamentaux

3 | 2004

Surveiller et punir / Surveiller ou punir ?

\title{
L'accès au droit et le droit au recours
}

Éric Péchillon

\section{OpenEdition}

Journals

Édition électronique

URL : https://journals.openedition.org/crdf/7481

DOI : $10.4000 /$ crdf.7481

ISSN : 2264-1246

Éditeur

Presses universitaires de Caen

Édition imprimée

Date de publication : 1 octobre 2004

Pagination : 49-60

ISBN : 2-84133-221-7

ISSN : $1634-8842$

Référence électronique

Éric Péchillon, "L'accès au droit et le droit au recours », Cahiers de la recherche sur les droits

fondamentaux [En ligne], 3 | 2004, mis en ligne le 18 décembre 2020, consulté le 14 novembre 2022

URL : http://journals.openedition.org/crdf/7481; DOI : https://doi.org/10.4000/crdf.7481 


\title{
L'accès au droit et le droit au recours
}

\author{
Éric PÉCHILLON
}

Maître de conférences à I'Université de Rennes I

\section{Les obstacles à la reconnaissance de droits effectifs au détenu}

A. La conceptualisation chaotique du droit du service public pénitentiaire

B. Les conséquences sur la qualification juridique du détenu

II. Les étapes préalables à la garantie du droit en prison

A. L'amélioration de l'accès au droit

B. L'ultime garantie du droit au recours contentieux

Pour traiter une telle question dans le cadre de ce colloque qui a choisi comme sous titre "Perspectives de la peine privative de liberté », il semble pertinent de reprendre la méthode mise en œuvre par la commission Canivet ${ }^{1}$ relative au contrôle extérieur des établissements pénitentiaires qui estimait que pour pouvoir préconiser un contrôle efficace, il faut avant tout connaître la matière à contrôler et être en mesure d'en définir un contenu prévisible. De la même manière, pour pouvoir définir des droits effectifs au détenu, il est indispensable de poser distinctement les données du problème. La question du respect des droits de l'individu durant la période de détention est une préoccupation relativement nouvelle qui va à l'encontre du mode traditionnel de détermination de la norme en prison.

Il n'est pas excessif de considérer que l'accès au droit et à la justice contient tous les autres droits. Il leur donne une effectivité. Le Conseil constitutionnel a d'ailleurs pris une décision en ce sens lorsqu'il a affirmé ${ }^{2}$ que si l'on admet qu'une loi qui confère un droit ne l'assortisse pas d'un recours effectif devant un juge, la garantie des droits n'est plus assurée et en application de l'article 16 de la Déclaration des droits de l'homme de 1789, il n'y a «plus de Constitution». L'élaboration d'une norme effective passe donc par un débat sur l'équilibre entre le pouvoir administratif et juridictionnel. L'accès au droit et à la justice devient un droit subjectif directement rattaché à celui de citoyenneté.

Deux approches de l'accès au droit sont possibles. La première vise à pouvoir avoir une connaissance suffisante des règles opposables à un individu dans une situation donnée, afin qu'il soit en mesure de comprendre les décisions prises à son encontre et éventuellement les contester devant une instance capable de les réformer. La seconde est plus large puisqu'elle vise à faire en sorte que l'ensemble des règles de droit soit suffisamment précis et prévisible pour pouvoir connaître les obligations réciproques de chacun des intervenants. Ces deux approches ne sont pas antinomiques mais complémentaires.

\section{Les obstacles à la reconnaissance de droits effectifs au détenu}

La prison a longtemps souffert du désintérêt du législateur concernant la prise en charge de la population 
privée de liberté à la suite d'une décision de justice. Ce désintérêt diminue progressivement, mais l'analyse du phénomène carcéral reste trop souvent dépendante d'analyses ponctuelles et conjoncturelles. Qu'en est-il du droit public et plus précisément du droit administratif? L'évolution du droit pénitentiaire public peut être illustrée au travers de trois textes symboliques.

\section{A. La conceptualisation chaotique du droit du service public pénitentiaire}

Au départ, l'Administration pénitentiaire est ignorée par les grands mouvements de réforme comme le montre le décret du 28 novembre $1983^{3}$. Ce texte a écarté sans motif valable l'ensemble des services du ministère de la Justice des règles du droit administratif général et conduit à l'isolement malencontreux de l'Administration pénitentiaire.

Ensuite, ce sont des réformes conjoncturelles qui aboutissent à reconnaître indirectement une place aux services pénitentiaires parmi les grands services de l'État ${ }^{4}$. Le contexte du débat sur la privatisation a permis de poser dans la loi le principe d'un service public administratif. L'article $1^{\text {er }}$ de la loi énonce que « Le service public pénitentiaire participe à l'exécution des décisions et sentences pénales et au maintien de la sécurité publique. Il favorise la réinsertion sociale des personnes qui lui sont confiées par l'autorité judiciaire. Il est organisé de manière à assurer l'individualisation des peines ».

Les conséquences de ce texte sont multiples ${ }^{5}$ :

- la qualification de service public pénitentiaire qui ne semble pas totalement privatisable compte tenu de certains missions régaliennes (direction et tenue du greffe)

- des missions antinomiques et hiérarchisées qui mette à la charge des services une obligation de résultat en matière de sécurité " extérieure » (lutte contre les évasions) et de moyens pour le reste;

- la reconnaissance implicite de l'applicabilité du droit administratif en fonction des particularités du service;

- le détenu usager du service public.

Enfin, c'est par une réforme générale des relations entre les citoyens et l'administration que le service public pénitentiaire s'est brutalement retrouvé confronté à l'application non seulement du droit administratif général mais dans une version modernisée. La loi du 12 avril $2000^{6}$ entraîne une multitude de modifications juridiques qui rendent indispensable une redéfinition des rapports de droits en détention ${ }^{7}$ :

- l'application du droit «commun » en prison;
- le détenu citoyen;

- l'affirmation de nouvelles missions à la charge de la puissance publique.

L'article 2 de cette loi a posé un «droit de toute personne à l'information qui garantit la liberté d'accès aux règles de droits applicables aux citoyens ». Pour donner une nouvelle dimension à l'adage «nul n'est censé ignorer la loi », le législateur a modifié le rapport de force en inversant la logique traditionnelle. Désormais, « les autorités administratives sont tenues d'organiser un accès simple aux règles de droit qu'elles édictent». «La mise à disposition et la diffusion des textes juridiques constituent une mission de service public au bon accomplissement de laquelle il appartient aux autorités administratives de veiller». Bien que la question ait été posée durant les débats parlementaires, cela ne va pas jusqu'à organiser une procédure d'information préalable et énonce encore moins un principe de participation des administrés à la préparation des textes réglementaires. Celle-ci n'est néanmoins pas exclue. Le législateur rappelle que cette mission est nécessaire à l'exercice de la citoyenneté.

En prison, l'accès matériel à la règle de droit pose un certain nombre de problèmes concrets allant à l'encontre de la culture pénitentiaire. Beaucoup de règles ne sont pas publiées et, si elles le sont, la consultation de bornes internet est envisagée avec méfiance par les différents services. La conséquence de cette situation est claire puisqu'elle aboutit de fait à une rupture d'égalité entre les citoyens en ce qui concerne l'accès à la norme.

Le travail du législateur procède d'une volonté d'aboutir à l'élaboration progressive d'un droit commun à l'ensemble des services publics au travers du choix affirmé de renforcer les droits des citoyens. L'émergence d'un statut applicable à l'ensemble des citoyens dans leurs relations avec les services publics administratifs montre qu'il est parfaitement possible de définir un droit applicable au public quelles que soient les raisons pour lesquelles il est en relation avec l'administration. En unifiant les règles applicables à l'ensemble des services publics administratifs, le législateur renforce la place des droits fondamentaux de l'individu. Conformément à la jurisprudence constitutionnelle, il affirme clairement que ces derniers ne peuvent être minorés par le pouvoir réglementaire. Un mouvement analogue de respect du droit commun à la fois procédural et conventionnel s'est manifesté en droit de l'application des peines, d'abord avec la loi du 15 juin 2000 dite "présomption d'innocence », puis dans le cadre des débats parlementaires précédant le vote de la loi portant adaptation de la justice aux évolutions de la criminalité.

\footnotetext{
3. Décret 83-1025 du 28 novembre 1983 concernant les relations entre l'administration et les usagers.

4. Loi 87-432 du 22 juin 1987 relative au service public pénitentiaire, JORF, 23 juin 1987, p. 6775.

5. É. Péchillon, Sécurité et Droit du service public pénitentiaire, Paris, LGDJ (Bibliothèque de droit public; t. 204), 1998 (avant-propos de G. Azibert, préface de F. Chauvin).

6. Loi n' 2000-321, JORF, 13 avril 2000, p. 5646.

7. M. Herzog-Evans et É. Péchillon, «L'entrée des avocats en prison et autres conséquences induites par la loi $\mathrm{n}^{\circ} 2000-321$ du 12 avril 2000 », D., 14 septembre 2000, "Chroniques », p. 481-484; Id., «Droit pénitentiaire : la réécriture de la loi par voie de circulaires ", Les Petites Affiches, $\mathrm{n}^{\circ} 56$, 20 mars 2001, p. 8-13.
} 


\section{B. Les conséquences sur la qualification juridique du détenu}

\section{Les quatre dimensions du statut juridique du détenu ${ }^{8}$}

Une personne incarcérée est, et demeure en toutes circonstances, une "personne humaine». L'État est par conséquent soumis à des obligations positives visant à garantir, en toutes circonstances, le respect des droits fondamentaux individuels. L'importance croissante de la jurisprudence de la Convention européenne de sauvegarde des droits de l'homme vient ici remettre en cause l'approche traditionnelle du rapport à l'État.

À un deuxième niveau, une personne incarcérée demeure un « citoyen ». La prison est une privation de la liberté d'aller et de venir, pas une éviction. Le rapport à l'État doit être appréhendé de la même manière qu'à l'extérieur. Reconnaitre le statut de citoyen n'est pas anodin car cela induit diverses conséquences juridiques dont la plus novatrice est le «droit à l'insertion» ${ }^{9}$. Ce droit fondamental à l'insertion va progressivement changer les obligations de la puissance publique envers les populations les plus en difficulté, dont les détenus font incontestablement partie. Ce droit n'est pas véritablement un "droit-créance », mais comme le soulignent certains un droit à la « reconnaissance ${ }^{10}$. Ce terme d'insertion consiste à faire entrer un individu dans une sphère dont il ne devrait pas être exclu. En lui donnant une portée juridique, la notion implique une démarche active de la puissance publique, une contrainte à mettre en œuvre une politique publique finalisée. Un auteur comme D. Lochak a bien mis en lumière cette tendance nouvelle du droit qui navigue entre normalité et normativité ${ }^{11}$. Les principes juridiques et les règles de droit varient en fonction de leur usage social. Dans certaines situations, le droit inscrit comme principe juridique ce qui est considéré comme normal et vise alors à développer des instruments capables d'offrir aux populations concernées les moyens d'y parvenir. Dans d'autres domaines, le principe posé tend vers un idéal. Les règles définies ont alors pour finalité d'orienter l'action des pouvoirs publics. La loi du 12 avril 2000 s'inscrit dans ce double mouvement. Elle consacre pour le détenu (comme pour l'ensemble de la population) un droit d'accès à la citoyenneté. Ce faisant, le législateur pose l'insertion comme le corollaire de la citoyenneté, ce qui passe par la reconnaissance de droits précis parmi lesquels l'accès au droit et le droit au recours.

De ce point de vue, cette affirmation de la citoyenneté du détenu était déjà présente en germe dans des textes législatifs généraux antérieurs. Par exemple la loi de 1988 relative à la lutte contre les exclusions consacrait un titre à « l'exercice de la citoyenneté ${ }^{12}$ en faisant du droit à l'insertion une obligation concrète à la charge de l'État. L'objectif du législateur est sans ambiguïté. Il faut parvenir à faire prendre part à la vie sociale des populations qui, compte tenu de la précarité dans laquelle elles se trouvent, ne peuvent y accéder. Pour faciliter une meilleure jouissance de leur droit, il est nécessaire de prévoir à leur égard des mesures de discrimination positives. Cela passe par l'octroi facilité d'une assistance juridique grâce à l'octroi de l'aide juridictionnelle ou le droit pour les détenus d'être informé sur leur droits sociaux. Depuis quelques années, la citoyenneté passe en partie par un accès à la justice et la bonne connaissance des règles de droit est un élément indispensable à la participation à la vie sociale.

À un troisième niveau, une personne incarcérée demeure un "justiciable» bénéficiant des droits procéduraux (principe du contradictoire, droit au recours juridictionnel) normalement prévus dans les matières considérées. Le prévenu comme le condamné sont en effet toujours en relation singulière avec le service public de la justice dont l'activité ne se limite pas au prononcé de la sanction. De par sa position originale dans l'organisation institutionnelle, le droit de la prison, en effet, traite de questions de nature juridique mixte, concernant simultanément le droit administratif, le droit pénal, le droit civil ou le droit du travail. Les garanties organisées dans ces disciplines doivent trouver à s'appliquer de manière cohérente à l'égard des détenus.

À un quatrième niveau, une personne incarcérée peut être qualifiée comme un « usager » étant en relation avec un service public administratif. Il en résulte que les détenus peuvent se prévaloir d'un droit à un fonctionnement normal du service à leur égard et à la mise en œuvre des missions fixées par la loi à la puissance publique.

Toutefois, se pose encore la question de savoir si la prison constitue un service public unique ou si au contraire il est possible de distinguer des services différents en fonction des catégories d'établissement: maison d'arrêt et établissement pour peines (voire plus précis en fonction du régime de l'établissement).

Ces quatre degrés dans la qualification du rapport à la puissance publique ne doivent en aucune façon être inversés. Jusqu'à présent, l'élaboration de la norme applicable durant la détention se faisait selon une logique inverse à celle-ci. Beaucoup trop souvent, l'Administration pénitentiaire fait prévaloir ses nécessités propres lorsqu'elle est amenée à édicter des règles. Dans ces conditions, le droit applicable à la prison ne prend pas suffisamment en compte les impératifs essentiels de protection des libertés fondamentales de l'individu. En conséquence de quoi, les dispositions édictées dans l'intérêt du service s'intercalent trop souvent entre les normes de rang supérieur et les situations qu'elles ont vocation à régir. La personne privée de sa liberté d'aller et de venir se trouve presque

8. L'importance de ces quatre points a d'ailleurs été réaffirmée récemment par l'assemblée plénière de la Commission nationale consultative des droits

de l'homme dans son «étude sur les droits de l'homme dans la prison» adoptée le 11 mars 2004.

9. É. Péchillon, «Le département et l'insertion, un responsable unique et une multitude de partenaires», Bulletin juridique des collectivités locales, avril 2004.

10. J.-P. Fitoussi et P. Rosanvallon, Le Nouvel Âge des inégalités, Paris, Seuil, 1996, p. 210.

11. D. Lochak, «Le droit, du normatif à la normalisation », in Le Sujet de droit, Ramonville, Érès, 1988, p. 127-145.

12. Articles 78 à 82 de la loi. 
toujours dépourvue de droits effectifs faute d'effets contraignants de la «norme » pour les services de l'État.

\section{Quel est le droit qui lui est applicable en détention?}

Les principales remarques que l'on peut faire sur ce droit sont désormais connues et peuvent être résumées autour de quelques points clés.

- Un droit dérogatoire qui subit les influences contradictoires d'une multitude de branches du droit: le droit pénal, le droit administratif et les finances publiques. La principale conséquence de cet enchevêtrement juridique est une confusion régulière dans l'emploi de notions juridiques comme:

- l'ordre public, l'ordre intérieur de l'établissement;

- la sécurité publique et la sécurité intérieure ;

- la dangerosité pénale, administrative ou médicale.

- Un droit inachevé qui ne respecte pas la hiérarchie des normes.

Le juge administratif reste d'ailleurs dans une situation délicate lorsqu'il s'agit de contrôler une demande d'abrogation d'une réglementation pénitentiaire ${ }^{13}$. Le respect du principe de légalité est au cœur de la réflexion sur la réforme du droit applicable durant la période de détention. C'est ainsi que l'Observatoire international des prisons demandait au Conseil d'État d'annuler la décision implicite du Premier Ministre refusant d'abroger le décret du 2 avril 1996 relatif au régime disciplinaire. Plusieurs moyens étaient soulevés tendant tous à contester la méthode employée pour aborder la norme en prison. La question centrale était de savoir si le Premier Ministre pouvait réglementer par décret des matières portant sur certains droits fondamentaux de l'individu (propriété, travail...). Le Conseil d'État fait ici une lecture linéaire du Code de procédure pénale et constate que trois articles de la partie législative du code (art. 720, 726 et 728) donnent à l'autorité administrative une compétence suffisante pour définir les modalités (fautes et sanctions) de la discipline en prison, d'autant plus que les mesures adoptées resteraient dans un cadre proportionné aux nécessités du service sans jamais dénaturer le cadre fixé par l'article 34 de la Constitution. Ensuite, le Conseil d'État la norme nationale au regard des exigences du droit international. Comme il a désormais pris l'habitude de le faire, il refuse de considérer la commission de discipline comme un tribunal et par conséquent écarte toutes les critiques basées sur une prétendue violation de l'article 6 de la CEDH. Cette approche française de la discipline au sein des services publics distincte de la matière pénale semble de plus en plus en décalage avec la jurisprudence européenne, mais strictement conforme à la logique de notre droit administratif. Néanmoins le Conseil d'État ne refuse pas par principe de confronter la norme interne aux contraintes internationales. En l'espèce, il estime par exemple que le sort réservé aux mineurs n'est pas contraire aux dispositions de l'article 3-1 de la Convention de New York du 26 janvier 1990, car si la France ne met pas en ouvre une procédure disciplinaire spécifique, elle organise néanmoins une information des représentants légaux et leur permet d'intervenir dans le débat disciplinaire contradictoire en présentant des observations. De la même façon, les sanctions prononcées, notamment les plus graves, tiennent compte de la minorité du détenu. Enfin, le Conseil d'État s'est prononcé plus précisément sur la sanction de mise en cellule disciplinaire. Sans surprise, le juge national considère que cette sanction limitée au maximum à 45 jours ne peut être dans son principe constitutive d'un traitement inhumain et dégradant (art. $3 \mathrm{CEDH}$ ). Au contraire, compte tenu du fait que les textes laissent au chef d'établissement la possibilité d'adapter la sanction à la gravité des fautes préalablement définies traduit bien la souplesse du mécanisme et sa proportionnalité. Par contre, en justifiant le fait qu'au nom « de la défense de l'ordre dans l'établissement et de la prévention des infractions pénales", le placement en cellule disciplinaire prive le détenu de son droit de visite, le Conseil d'État privilégie le fonctionnement du service public pénitentiaire au détriment des droits de l'individu énoncés à l'article 8 de la CEDH. Le caractère automatique de cette privation de droit et l'absence de lien avec les faits à l'origine de la sanction auraient sans doute mérité une analyse plus circonstanciée de la réalité carcérale.

- Un droit inaccessible : l'abus des circulaires et la prolifération des pratiques locales.

Constatant « la profusion de circulaires, instructions et notes de service» qui régissent le fonctionnement du service public pénitentiaire, le « rapport Canivet " ${ }^{14}$ parle d'un «droit subordonné» qui sert de base à l'action administrative. Or, en principe, les circulaires ne donnent aucun fondement juridique à un acte. Pourtant, elles ont une influence certaine sur l'action administrative, et à ce titre on peut considérer la circulaire comme source de légalité interne. Par conséquent, et même lorsque les circulaires traduisent une volonté d'encadrement du pouvoir de l'administration, cela n'est pas suffisant pour contrôler certaines mesures dont les conséquences à l'égard du détenu peuvent être particulièrement graves.

En principe, la circulaire doit rester purement interprétative. Elle ne sert qu'à expliciter, interpréter une norme juridique (loi ou règlement) préexistante et à orienter l'action d'un service. Malgré leur publication prévue par la loi du 17 juillet 1978, «dans les rapports entre l'administration et les administrés, elles sont comme si elles n'existaient pas; elles sont la transparence même ${ }^{15}$. Pour certains, il ne s'agit absolument pas d'un acte juridique. Il s'agit d'un document à caractère strictement administratif qui n'intéresse que les relations entre les agents et leurs supérieurs ${ }^{16}$. Parfois la circulaire va toutefois sortir de

13. CE 30 juillet 2003 OIP, section française, req. $\mathrm{n}^{\circ} 253973$.

14. Rapport sur l'«amélioration du contrôle extérieur des établissements pénitentiaires », p. 72 sq.

15. R. Chapus, Droit administratif, Paris, LGDJ, p. 513.

16. Y. Gaudemet, «Remarques à propos des circulaires administratives », in Mélanges Stassinopoulos, Paris, LGDJ, 1974, p. 561. 
son rôle et contenir des prescriptions juridiques, propres à fonder l'action administrative. Dans ce cas, la jurisprudence considère que la circulaire perd sa nature interprétative, elle va devenir réglementaire ${ }^{17}$. La circulaire sera considérée réglementaire lorsqu'elle sera, selon le terme de R. Chapus, «innovatoire». Il s'agira alors d'une véritable règle de droit, d'un règlement et sa légalité sera examinée comme pour tout autre règlement. De même, la circulaire réglementaire sera opposable aux administrés comme à l'administration, dans les conditions habituelles de tout acte réglementaire.

Ces principes s'appliquent au service public pénitentiaire comme ailleurs. Ainsi, une disposition d'une circulaire relative aux fouilles a été considérée par le Conseil d'État réglementaire et légale, tandis que dans le même arrêt, une disposition d'une autre circulaire relative à la correspondance, après avoir été considérée réglementaire, a été annulée au motif qu'elle restreignait de manière trop générale la liberté de correspondance des détenus ${ }^{18}$. De même, à propos de l'article 24 de la loi du 12 avril 2000 : la circulaire du 31 octobre 2000 avait mis en place une procédure d'agrément des personnes susceptibles d'assister le détenu. Le Conseil d'État a annulé la partie de la circulaire instituant cette procédure car le ministre de la Justice n'avait pas compétence pour poser de telles règles ${ }^{19}$. La loi renvoyait à un décret en Conseil d'État pour les modalités d'application. Par la suite, un tel décret a été pris, instituant légalement une procédure d'agrément ${ }^{20}$.

Mais l'application de la distinction entre circulaire réglementaire et interprétative est loin d'être évidente en pratique. Elle donne une vision réductrice et quelque peu simpliste de la réalité. En effet, peu de circulaires sont considérées réglementaires, alors qu'une telle portée pourrait leur être reconnue. Cela est dû d'une part à la relative faiblesse du nombre des recours dont font l'objet les circulaires, notamment en matière pénitentiaire, et d'autre part à l'attitude du Conseil d'État, peu enclin à reconnaître le caractère «innovatoire» des circulaires, car cela l'obligerait le plus souvent à annuler le texte $^{21}$. Or de nombreuses circulaires manifestent bien plus qu'un simple commentaire de règles préexistantes. Elles contiennent parfois une volonté normative. Dans la pratique administrative, les agents vont bien souvent se référer à la circulaire pour agir, ce qui confère à celle-ci une portée bien plus importante que celle que lui reconnaît l'état actuel du droit. D'ailleurs les circulaires suivent la voie du pouvoir hiérarchique, ce qui leur donne un caractère obligatoire à l'égard des agents. Enfin, l'action interprétative n'est pas neutre. Elle conditionne la façon dont la règle va être appliquée. Elle donne un sens à la règle. Cela conduit certains auteurs à reconnaître «la réalité normative des circulaires et instructions administratives ${ }^{22}$. Rivero avait vu dans les circulaires l'expression de la «légalité intérieure» des services publics ${ }^{23}$. En ce sens, on peut parler d'une légalité intérieure officieuse.

Certes, on peut objecter que si le droit nie la valeur juridique des circulaires, l'administré s'y retrouve à l'occasion de l'examen d'une décision individuelle et en invoquant les textes de la légalité générale. C'est d'ailleurs ce qu'il faut espérer à l'égard de l'application de la loi du 12 avril 2000 dans le domaine pénitentiaire. À l'occasion d'éventuels recours contre une décision individuelle ayant écarté l'application de l'article 24, le juge administratif pourra rectifier le tir en interprétant in concreto les circonstances au regard de la loi.

L'état actuel du droit laisse dans l'ombre des textes tels que les circulaires dont on ne peut nier l'impact. Il y a là une source potentielle importante d'incertitudes juridiques. D'autant que les circulaires foisonnent en droit pénitentiaire. Elles sont sans aucun doute nécessaires pour pallier les insuffisances de la loi et permettre de s'adapter aux circonstances, notamment aux conditions matérielles souvent précaires des établissements pénitentiaires. Toutefois, elles constituent une source de légalité officieuse qui ne permet pas de contrôler certaines mesures d'ordre intérieur.

\section{Les étapes préalables à la garantie du droit en prison}

Pour parvenir à une application efficiente du droit en détention, il est indispensable que le service public pénitentiaire ne soit plus présenté comme jouissant d'une position d'exception au regard d'autres secteurs ou activités de l'État. Cela passe par l'amélioration de l'accès matériel à la règle de droit, notamment au travers d'une réflexion sur le règlement intérieur $(\mathrm{A})$ mais aussi par la détermination précise de critères permettant de connaître avec précision le juge compétent pour examiner les requêtes (B).

\section{A. L'amélioration de l'accès au droit}

«Pivot d'un réseau d'informations à l'intérieur de chaque établissement», le règlement intérieur est fait pour rappeler « qu'être détenu signifie être confronté à de nombreuses règles de vie dérogatoires au droit commun

17. CE Ass., 29 janvier 1954, Institution Notre Dame du Kreisker, rec. p. 64; RPDA, 1954, p. 50, concl. B. Tricot; AJ, 1954, 2 bis, p. 5 , chronique F. Gazier et M. Long; GAJA, $\mathrm{n}^{\circ} 80$.

18. CE, 8 décembre 200o, Frérot, req. $\mathrm{n}^{\circ}$ 162-995, concl. R. Schwartz, $L P A, \mathrm{n}^{\circ} 28,8$ février 2001, p. 16.

19. CE, 20 mars 2002, Korber, dieng Lobat, Ordre des avocats à la Cour de Paris et autres, req. $\mathrm{n}^{\circ}$ 226-803, 227-452, 227-847, 227-848, 228-666 et 228-686.

20. Décret $\mathrm{n}^{\mathrm{0}}$ 2002-1023 du 25 juillet 2002 relatif à «l'application de l'article 24 de la loi $\mathrm{n}^{\circ}$ 2000-321 du 12 avril 2000 à l'Administration pénitentiaire et relatif aux mandataires susceptibles d'être choisis par les personnes détenues ", JORF, $\mathrm{n}^{\mathrm{o}} 178,1^{\mathrm{er}}$ août 2002, p. 13098.

21. R. Chapus, Droit administratif, p. 516.

22. P. Combeau, «Un oubli dans la réforme : l'invocabilité des circulaires et instructions administratives », AJDA, 2000, p. 495.

23. J. Rivero, op. cit., notamment p. 91 sq. 
en raison des contraintes inhérentes à la privation de liberté ${ }^{24}$. Pour l'administration centrale, la fonction de ce document paraît relativement claire, ce document contraignant devrait servir à asseoir l'autorité du personnel pénitentiaire sur la population pénale et sur toute personne pénétrant dans l'enceinte de l'établissement. Ce faisant, il s'agit de permettre d'adapter la réglementation nationale aux spécificités de chaque lieu de détention. La multiplication des règles locales est toutefois plus le résultat d'une incapacité à poser des principes généraux applicables sur l'ensemble du territoire qu'un véritable choix juridique ou une politique concertée de management.

Le droit pénitentiaire présente en effet la particularité d'avoir permis de renoncer à imposer des règles applicables à l'ensemble de la population carcérale. Si l'objectif était de prendre en considération le sort de chaque détenu, on pourrait peut-être en conclure que le service public pénitentiaire est parvenu à donner un sens positif à la privation de liberté ; mais sa logique est toute autre puisqu' elle permet de contourner les rares exigences posées par les règles générales en invoquant des motifs de sécurité ou des difficultés matérielles de transposition des grands principes (organisation architecturale des lieux, dangerosité de la population pénale...). Cette situation peut s'expliquer de différentes manières, notamment par l'attitude du juge administratif. Mais au-delà des causes, ce sont les conséquences de cette absence de qualification juridique qu'il faut prendre en considération. L'ensemble de la rigueur normative se trouve remis en cause.

Chaque lieu de détention génère son propre mode de fonctionnement en jouant sur la marge de manœuvre accordée par les ambiguïtés inscrites dans les articles du Code de procédure pénale. Le principal responsable de l'organisation locale se trouve être en pratique le chef d'établissement, sans que pour autant celui-ci ne dispose des véritables moyens juridiques et financiers de mener à bien ses missions. Son pouvoir discrétionnaire est d'autant plus fort qu'en plus de «fixer la loi » dans les murs, il est considéré comme l'interlocuteur privilégié des relations entre les détenus et l'administration. À travers le règlement intérieur, le directeur dispose d'un outil, d'un document «de référence» assurant son autorité sur la population pénale, et plus indirectement sur le personnel de surveillance. Légèrement standardisé dans sa présentation, ce texte permet de mettre en lumière la conception de la détention défendue par le directeur, car c'est à lui qu'il revient majoritairement d'orienter le fonctionnement de son établissement, dans les trop rares limites imposées par la catégorie du lieu qu'il dirige. La mise à jour régu- lière du document et sa diffusion sont des moments privilégiés pour affirmer la conception de l'État de droit dans l'enceinte de l'établissement. Il en est de même lorsque le document est délaissé par l'équipe de direction.

Cette conceptualisation relativement libre du règlement intérieur fait donc de l'échelon local un acteur puissant de la vie quotidienne en détention. Présenté soit comme une nécessité, soit comme une obligation traditionnelle de toute vie en collectivitée ${ }^{25}$, le règlement intérieur n'a pourtant toujours pas de véritable statut juridique. Aucun échelon de l'Administration pénitentiaire ne prend en effet le soin de l'expliciter. Le flou juridique qui entoure ce document n'est pourtant pas sans incidence.

\section{Sur sa rédaction}

Ce type de norme, touchant directement à la vie quotidienne des détenus, ne devrait plus pouvoir être élaboré unilatéralement; il relève au contraire de la catégorie des «règles négociables». L'élaboration d'une réglementation propre à chaque lieu de détention représente l'une des parties les plus délicates de son développement du droit pénitentiaire qui cherche encore à développer son assise locale et à préciser son contenu. La négociation peut s'envisager à différents degrés et selon des formes plus ou moins contraignantes. De par son contenu, le règlement intérieur concerne les personnels qui vont avoir à l'appliquer, mais aussi les collaborateurs, les visiteurs, les familles et les détenus qui vont devoir s'y conformer. Toutes les règles ont en effet une influence directe sur l'organisation et le fonctionnement du service.

Telle qu'elle est actuellement conçue, la procédure d'élaboration du règlement intérieur n'est pas véritablement satisfaisante car la concertation prévue reste largement fictive. L'efficacité des réunions de préparation destinées à recueillir les doléances et les souhaits des membres du personnel et des multiples intervenants extérieurs dépend trop largement de la personnalité du directeur. C'est encore plus le cas lorsque la directive évoque la participation des détenus au moyen de l'instauration « d'une méthode permettant aux intéressés d'exprimer leur point de vue sur l'aménagement du quotidien ${ }^{26}$. Si l'on peut admettre que le choix définitif et la finalisation du document reviennent au chef d'établissement, il faut déplorer un système dont la réussite dépende aussi étroitement de la personnalité d'un seul individu. Touchant directement à la vie quotidienne des détenus, ce type de norme ne doit plus rester dans le domaine de la pure décision unilatérale, mais progressivement passer dans la catégorie des « règles négociées » ${ }^{27}$.

24. Circulaire AP 88-16 G2 du 27 décembre 1988 relative aux règlements intérieurs des établissements pénitentiaires, Bull. off. Min. Justice, $\mathrm{n}^{\circ} 32$.

25. La formule suivante est d'ailleurs très souvent inscrite en préambule du document: «Toute vie en collectivité implique des règles à respecter. La vie en détention est pour sa part, caractérisée par des règles de vie dérogatoire au droit commun en raison des contraintes inhérentes à la privation de liberté. Aussi, plus que dans toute autre collectivité, la vie dans un établissement pénitentiaire, compte tenu du fait qu'elle est imposée, qu'elle se déroule dans un espace clos et restreint, nécessite le strict respect par chacun et dans l'intérêt de tous, des règles de vie fondées sur des exigences de discipline.

26. Circulaire AP 88-16 G2 du 27 décembre 1988, p. 118-119 (cf. note 24).

27. Le Code de procédure pénale doit contenir précisément les domaines adaptables à chaque lieu de détention et poser une procédure de négociation et de contrôle du contenu des règlements. 
Les différents avis rendus à la suite de toute modification doivent certes être adressés à diverses autorités (juge de l'application des peines, directeur régional), ce qui ne suffit pas à mettre en place un véritable contrôle préventif de la légalité de son contenu. À l'heure où l'on commence à parler de la mise en place d'une instance extérieure de régulation et de contrôle de l'activité pénitentiaire, il est important de rappeler que la transparence administrative et le respect de l'État de droit doivent aussi passer par la certitude que des documents aussi contraignants pour la vie quotidienne ne peuvent pas contenir des mesures contraires à la légalité générale et surtout pas inutilement limitatives de droits fondamentaux de la personne humaine. Par contre, toutes les dispositions du règlement intérieur ne sont pas négociables, ni même adaptables. De manière parfaitement justifiée, l'Inspection générale des services n'autorise aucun assouplissement ni aménagement sur les textes relatifs à la sécurité au sens strict. L'exemple le plus significatif est l'organisation de l'usage des armes.

Le règlement intérieur doit donc tendre à devenir une œuvre de concertation plus que l'expression du pouvoir discrétionnaire du chef d'établissement. Sur ce point, il faut noter que le texte du document est communiqué pour avis au juge de l'application des peines, et transmis au directeur régional des services pénitentiaires pour approbation $^{28}$. En pratique, nous avons constaté que cet échange n'est pas toujours fructueux comte tenu notamment de la différence de culture des deux intervenants. Ainsi l'appréciation des exigences de légalité varie-t-elle considérablement en fonction de l'origine de l'interlocuteur (par exemple de l'importance de sa formation juridique en droit public). Le chef d'établissement, en plus de son influence en matière de détermination des règles quotidiennes de fonctionnement, est un interlocuteur obligé pour tous les intervenants. Il doit donc perpétuellement ménager les «susceptibilités » des autorités dont dépend largement le fonctionnement quotidien de son établissement. Il ne possède certes pas une totale liberté dans le choix de ses partenaires, mais c'est de ses qualités personnelles et de son sens de l'organisation que dépendent largement les relations de son service avec l'extérieur. Cet aspect de ses fonctions s'avère d'autant plus crucial que l'autorité judiciaire et les défenseurs du détenu forment la catégorie des contacts obligatoires, que l'Administration pénitentiaire est tenue de mettre en œuvre, et même de faciliter.

Le règlement intérieur contribue directement à la préservation du fragile équilibre carcéral. Aussi doit-il être régulièrement révisé pour tenir compte du fait que «l'évolution des mœurs exerce aussi son influence à l'intérieur des murs des prisons ${ }^{29}$, mais aussi de l'évolution rapide des réglementations les plus diverses ${ }^{30}$. La procédure de sa révision, comme celle de son élaboration, ne doit pas rester le fruit de la volonté unique du chef d'établissement. S'il est juridiquement l'auteur du texte, et si pratiquement chaque lieu de détention possède un règlement proche des positions de son directeur ${ }^{31}$, il ne faut pas perdre de vue que les règles ainsi définies ne disposent que d'une autonomie limitée. Elles doivent par exemple respecter, dans la mesure du possible, le principe d'égalité des usagers devant le service public au sein d'un même lieu d'incarcération. Les règles principales de fonctionnement ne sont théoriquement pas adaptables. Toutefois, certaines d'entre elles bénéficient d'un relatif degré de tolérance quant à leur mise en œuvre au sein des divers établissements. Les détenus d'un même lieu sont traités de manière identique (du moins, cela est inscrit dans les textes); par contre, la liberté d'élaboration et de révision des règlements intérieurs entraîne des traitements variables en fonction de la prison dans laquelle le détenu est incarcéré. Ce décalage de traitement n'est pas toujours bien compris par les personnels et les détenus qui sans avoir changé de statut ou de catégorie d'établissements se voient opposés des normes différentes. L'interdiction de posséder une console de jeu vidéo, une armoire est souvent mal vécue lorsque l'objet à été préalablement cantiné. L'usage de la norme est ici directement lié au chef d'établissement qui se sert du règlement intérieur pour inscrire dans un document les contraintes de son établissement (installation électrique, nuisances sonores...), les particularités de son lieu d'implantation (offre de services extérieurs comme la formation ou la religion). Certains directeurs ont en effet fait le choix de consigner dans le règlement la carence d'offre extérieure pour justifier le traitement dérogatoire subi par les détenus. Au moins au moment de sa révision, le document sert également de moyen de communication interne pour insister sur les normes subies (à savoir celles qui s'imposent à l'Administration pénitentiaire comme à n'importe quel autre service) ou pour marquer la volonté du directeur de mener une politique locale (sécuritaire ou non d'ailleurs).

\section{Sur son contenu}

Il faut mentionner une difficulté liée au fait que le règlement intérieur ne contient pas toutes les règles relatives à la vie de l'établissement. En effet, le directeur d'établissement a la possibilité de fixer des règles de conduite collective en dehors de ce document. Il use ainsi souvent de notes de service et d'instructions ponctuelles, dont la variété et le caractère pointilleux ne doivent pas servir à cacher leur véritable force contraignante. Le Conseil d'État a d'ailleurs nettement admis que ce type de dispositions constituait des actes juridiques contestables devant le juge ${ }^{32}$. Dans cette affaire, il s'agissait du choix des produits de la cantine et du mode de gestion de celleci (notamment les produits informatiques).

28. À défaut de réponse de sa part dans le délai d’un mois, le règlement intérieur est réputé agréé. Agréé ou réputé tel, le directeur régional adresse un exemplaire du règlement intérieur ou de toute modification à l'administration centrale.

29. M. Leteneur, «La discipline pénitentiaire», RPDP, 1984, p. 235

30. Droit administratif général, procédure pénale, Droit de la sécurité sociale et de la santé..

31. L'absence ou le caractère obsolète du document constitue sur ce point un élément intéressant du rapport au droit au sein d'un établissement.

32. CE 18 mars 1998 Druelle, notamment l'analyse faite par le commissaire du gouvernement Bonichot, RFDA, 1999, p. 227. 
Néanmoins, tout établissement carcéral devrait posséder son propre règlement intérieur destiné à fixer les particularités de son régime de fonctionnement. Ce document est l'expression, légèrement standardisée dans sa présentation, du droit applicable au sein de l'établissement. Il exprime largement la conception de la détention défendue par le directeur, car c'est ce dernier qui détermine majoritairement l'orientation du fonctionnement de son établissement, dans les grandes limites fixées par la catégorie de l'établissement qu'il dirige. D'un point de vue formel, le règlement doit contenir un certain nombre de rubriques obligatoires ${ }^{33}$. Néanmoins, les diverses tentatives d'uniformisation du fonctionnement des établissements se heurtent aux particularités locales ${ }^{34}$. La réalisation d'un tel document doit par conséquent être la conjugaison des dispositions nationales et des spécificités de chaque lieu de détention. Quelle que soit sa rédaction, il ne peut jamais se résumer à un document uniformisé.

Lorsque l'on s'arrête sur le contenu du règlement intérieur, la première chose qui frappe le visiteur est sa taille. Le volume du document s'explique en grande partie par le fait que jusqu'à présent la fonction de ce document n'est pas définie de manière cohérente. Pour l'essentiel, il se contente souvent de reprendre les dispositions du Code de procédure pénale qui s'applique dans l'établissement. Cette reprise ne s'impose pourtant pas et alourdit considérablement la mise à jour du document. S’il doit servir de référence à chacun, cela ne sous-entend pas qu'il doive contenir l'intégralité des règles en vigueur. Sinon pourquoi se contenter d'indiquer celles issues d'un seul code? Si sa rédaction consiste à fournir un accès au droit, elle ne doit pas uniquement être pensée sous un angle disciplinaire.

\section{Sur son autorité}

Selon l'administration centrale, il devrait représenter le document majeur de la vie carcérale, puisqu'il est à la fois l'expression de l'autorité du chef d'établissement, et le document de référence aussi bien pour les détenus que pour le personnel. Dans la communauté carcérale, il constitue l'instrument conférant conjointement l'autorité et la légitimité aux décisions des responsables de la surveillance, auprès d'une population pénale qui manque souvent de repères normatifs. Juridiquement, le règlement intérieur de l'établissement de détention constitue un acte administratif faisant grief. Cette qualification entraîne un certain nombre de conséquences :

- Toute personne concernée par son contenu (détenu, famille, avocat, visiteur) peut contraindre l'administration à le modifier ou à abroger l'ensemble des dispositions illégales.

- Le recours pour excès de pouvoir devant le Tribunal administratif est possible afin d'obtenir l'annulation des articles non conformes au droit en vigueur. Sur ce point, le juge administratif commence à approfondir son contrôle de la légalité. Il examine quelle est précisément la part de liberté d'interprétation dont dispose le chef d'établissement lorsqu'il fixe une règle contraignante. Les détenus peuvent donc utilement revendiquer devant la justice l'existence de leurs droits et contraindre l'administration à fournir les prestations légales. Mentionnons à titre d'exemple l'article 450 du CPP qui est maintenant considéré comme générant une obligation pour l'administration en matière de resocialisation. À chaque fois que le code emploie le verbe «devoir» à l'encontre de l'autorité publique, le juge aura tendance à durcir son contrôle avec un examen dit de "proportionnalité» entre l'ordre et la sécurité qui doivent être maintenus « avec fermeté » mais «sans apporter plus de contraintes qu'il n'est nécessaire pour le maintien de la sécurité et d'une bonne organisation de la vie en collectivité ${ }^{35}$.

- La commission d'accès aux documents administratifs a affirmé que tout règlement intérieur était communicable de plein droit à toute personne qui en ferait la demande ${ }^{36}$.

Se pose donc rapidement la question de la standardisation des règlements intérieurs ${ }^{37}$. Or, jusqu'à présent, les diverses tentatives d'uniformisation sont restées sans effet. Il faut tenir compte du fait qu'en pratique pour des raisons multiples tenant notamment à la personnalité du chef d'établissement ou à la configuration matérielle des lieux, les détenus ne sont pas tous traités de la même manière en fonction de leur lieu de détention et ceci, alors même qu'ils appartiennent à la même catégorie juridique (prévenus, condamnés à des courtes peines ou à perpétuité...). Le principe juridique d'égalité des usagers à la base du droit des services publics n'est pas respecté dans les prisons françaises. Rappelons que pour pouvoir légalement mettre en place un traitement différent, l'autorité administrative doit réussir à démontrer que la population à laquelle le texte s'adresse est placée dans une situation juridique spécifique et objectivement différentiable (ce qui n'est, la plupart du temps, pas le cas).

La fonction du règlement intérieur est à strictement parler d'organiser la vie en collectivité dans son déroulement quotidien. Pourtant, la standardisation minimum du contenu du texte remis aux détenus impose l'incorporation de l'ensemble de ce qu'il est possible d'appeler

33. Selon la circulaire AP 88-16 G2 du 27 décembre 1988 JUSE884007oC, ces rubriques sont : l'emploi du temps, les relations du détenu, l'orientation et le transfèrement, l'isolement, la gestion des valeurs pécuniaires, les activités, les soins, l'assistance spirituelle ou morale, le service socio-éducatif, les questions d'habillement, d'hygiène et d'entretien, et enfin, la discipline (curieusement, c'est la dernière rubrique obligatoire).

34. Les deux principales sont d'une part, l'organisation matérielle des locaux qui constitue souvent une contrainte importante car elle ne peut pas toujours s'adapter aux réformes réglementaires; d'autre part, l'origine et la personnalité de chacun des détenus composant les usagers d'un établissement. Le même établissement sera en effet amené à modifier son fonctionnement si le traitement de certaines situations difficiles l'impose, comme une hausse du nombre de toxicomanes.

35. Cette position, relative à l'interprétation de l'article D $242 \mathrm{CPP}$, est celle défendue par M. Bonichot devant le Conseil d'État à propos de l'affaire Druelle. 36. Article 2 de la loi du 17 juillet 1978. Mentionnons toutefois que celui-ci pourra être facturé aux demandeurs.

37. Cette question de l'uniformisation ou de l'harmonisation des règlements intérieurs date de la fin des années soixante-dix et ne semble pas encore totalement abandonnée par la direction de l'Administration pénitentiaire. 
les grands principes du droit pénitentiaire. On retrouve donc, dans ce document, toutes les difficultés d'un tel service public. Chaque règlement intérieur comporte ainsi un préambule, dans lequel est inscrit "qu'être détenu signifie être confronté à de nombreuses règles de vie dérogatoires au droit commun en raison des contraintes inhérentes à la privation de liberté ${ }^{38}$. Cette nécessaire mise en garde a pour fonction de placer le détenu en situation de sujétion vis-à-vis de l'autorité administrative. Par contre, le reste du document devrait être conçu pour fixer le cadre légal à respecter par les agents et les usagers du service dans l'organisation des droits et prérogatives de chacun.

\section{B. L'ultime garantie du droit au recours contentieux}

«L'exécution des jugements répressifs peut paraître directement liée à l'exercice de la fonction juridictionnelle, dont elle est la conséquence. Le juge administratif a initialement souscrit à une telle filiation, le conduisant à une interprétation restrictive de sa compétence. Les critères plus récemment dégagés ont sensiblement assoupli cette jurisprudence ${ }^{39}$. Au départ le Conseil d'État a surtout donné une définition négative de sa compétence ${ }^{40}$, refusant d'admettre sa compétence pour examiner les demandes fondées sur «les conditions d'exécution des peines ${ }^{41}$ ou sur le régime de la détention préventive ${ }^{42}$. Pourtant, "il s'agit d'un service public typiquement administratif de gestion public, mettant en œuvre une des prérogatives les plus importantes et les plus traditionnelles de l'État. Cet élément paraît incontestablement appeler la compétence du juge administratif ${ }^{43}$.

Il faut attendre 1952 pour que la position du juge administratif devienne véritablement construite. Le Conseil d'État commence alors à procéder à une distinction entre les divers actes d'exécution des jugements. Sa méthode est surtout pragmatique et dépend surtout des affaires qu'il reçoit ${ }^{44}$. Il n'existe pas vraiment de critère objectif de partage des compétences. Le juge administratif l'admet sans la justifier. La jurisprudence semble cependant reconnaître la nécessité de la présence du juge administratif, là où le juge judiciaire, faute de texte, n'aurait certainement pas accepté de connaître un tel litige : c'est manifestement le cas en matière de responsabilité de la puissance publique.

Le refus d'examiner les décisions relevant de l'exécution de la «mission judiciaire » va trouver une remarquable application ${ }^{45}$. Monsieur Fargeaud d'Epied a été condamné à une peine de travaux forcés. Après sa condamnation, il est resté dans un centre d'internement administratif sans être transféré. Il a été assassiné. Sa veuve veut que l'État soit condamné à lui payer une indemnité en réparation des fautes commises par l'administration. Saisi d'un conflit négatif le Tribunal des conflits va prendre une position de principe toujours en vigueur. Puisque le Conseil d'État ${ }^{46}$ estimait qu'il n'avait pas à connaître les conditions d'exécution d'une peine prononcée par une juridiction judiciaire et que le juge judiciaire ${ }^{47}$ considérait pour sa part qu'une action fondée sur une méconnaissance par l'administration de la législation relative aux travaux forcés relevait de la compétence administrative ${ }^{48}$ car se situant dans la phase administrative de l'exécution des peines, le Tribunal des conflits a admis la compétence administrative pour apprécier la légalité des actes qui se rattachent directement au fonctionnement administratif des services pénitentiaires: "Considérant que s'il n'appartient qu'à l'autorité judiciaire de connaître des litiges relatifs à la nature et aux limites d'une peine infligée par une juridiction judiciaire et dont l'exécution est poursuivie à la diligence du ministère public, un litige relatif au retard apporté à transférer un condamné aux travaux forcés dans une maison de force intéresse le fonctionnement administratif du service pénitentiaire, lequel échappe au contrôle des magistrats de l'ordre judiciaire et ne relève que de la juridiction administrative $»{ }^{49}$.

Le Tribunal des conflits persiste à maintenir cette distinction pourtant datée et discutable ${ }^{50}$. Le juge administratif est donc le seul compétent pour examiner les conséquences dommageables résultant d'une permission

38. Circulaire AP 88-16 G2 du 27 décembre 1988.

39. S. Regourd, «Contrôle contentieux et administration pénitentiaire», D., 1983, p. 598

40. CE 27 février 1920 Mahine, Rec. p. 227.

41. CE 23 juillet 1920, Valette, Rec. p. 738 (dans cette affaire, Valette conteste la décision du ministre de le maintenir en détention, alors qu'il estime avoir accompli sa peine et que les 5 ans de résidence auquel il est astreint n'autorisent pas son maintien en détention).

42. CE 6 mars 1935, Brunaux, Rec. p. 295.

43. J.-M. Auby, «Le contentieux du service public pénitentiaire», RDP, 1987, p. 552.

44. TC 27 novembre 1952, Préfet de la Guyane, Rec. 642 : "Considérant que les actes incriminés sont relatifs, non à l'exercice de la fonction juridictionnelle, mais à l'organisation même du service public de la justice; que l'action des requérants a pour cause le défaut de constitution des tribunaux de première instance et d'appel dans le ressort de la Guyane, résultant du fait que le gouvernement n'a pas pourvu effectivement ces juridictions des magistrats qu'elles comportaient normalement; qu'elle met en jeu la responsabilité du service public indépendamment de toute appréciation à porter sur la marche même des services judiciaires; qu'il appartient dès lors à la juridiction administrative d'en connaître. »

45. TC 22 février 1960, Fargeaud d'Epied, Rec. p. 855.

46. CE 15 février 1952, Fargeaud d'Epied, Rec. p. 111.

47. Tribunal civil de Gap 2 février 1956, inédit.

48. Décret du 17 juin 1938. La peine doit être subie dans une maison de force.

49. Position régulièrement confirmée par la suite, TC 4 juillet 1983 Caillol, Rec. p. 541. Un prévenu en maison d'arrêt conteste la décision du chef d'établissement qui le place en quartier de haute sécurité. «Considérant que le litige auquel peut donner lieu une telle mesure, concernant un inculpé prise par une autorité administrative, et sans relation avec les nécessités de l'instruction, intéresse le fonctionnement administratif pénitentiaire et relève dès lors de la compétence de la juridiction administrative».

50. TC 3 juillet 2000, Garde des Sceaux c. Consorts Primau et Fosset, req. ${ }^{\circ} 3198$. 
de sortir. C'est donc à lui qu'il revient d'engager la responsabilité sans faute de l'État sur le fondement du risque ${ }^{51}$. La solution n'est pas évidente et le législateur devrait réfléchir à l'éventuelle désignation d'une unique autorité juridictionnelle compétente pour juger l'ensemble de la question pénitentiaire et disposant d'outils d'analyse adaptés à la réalité pénitentiaire. Depuis la loi « présomption d'innocence ${ }^{52}$, le législateur vient régulièrement juridictionnaliser ${ }^{53}$ la plupart des décisions ${ }^{54}$ du juge de l'application des peines, sans que cela ne conduise le Tribunal des conflits à revoir ses critères d'analyse. Le Garde des Sceaux prétendait que la juridiction de l'ordre judiciaire devait être déclarée compétente pour connaître le litige étant donné qu'il ne pouvait s'agir d'un litige se rattachant au fonctionnement administratif du service public pénitentiaire. Le Conseil d'État a préféré renvoyer l'affaire devant le Tribunal des conflits afin qu'une position claire soit adoptée. La solution adoptée, qui veut que le juge administratif conserve le monopole de sa compétence pour examiner les conséquences dommageables d'une permission de sortir, est faussement simple.

À la suite de cette affaire, il apparaît que le contentieux de la responsabilité sans faute de l'État résultant «d'un crime ou d'un délit » postérieur à une mesure libérale de traitement accordée par un juge de l'application des peines reste du ressort du juge administratif. Ce choix présente l'avantage d'unifier un contentieux et permettra à un seul ordre de juridiction de développer des critères cohérents d'analyse des activités liées à la détention. En plus de la continuité ${ }^{55}$, cette décision du Tribunal des conflits présente l'avantage de la simplicité, bien qu'elle ne réponde toujours pas à la question de la qualification du droit de l'exécution des peines.

Peut-on alors valablement reprendre l'argumentation classique défendue par certains commissaires du gouvernement selon laquelle « la faute des services pénitentiaires ne constitue pas une faute des services judiciaires, ni ne se rattache au fonctionnement de la justice ${ }^{56}$ ou que les accidents «ne sont liés ni dans leur cause, ni dans leur développement au fonctionnement du service judiciaire ${ }^{57}$ ? Doit-on toujours considérer que ce type de décisions se rattache forcément à l'exécution des peines?

La solution adoptée va à l'encontre de la jurisprudence Giry ${ }^{58}$ et des dispositions de la loi du 5 juillet 1972 qui pose le principe que le juge judiciaire est compétent pour connaître des conséquences dommageables résultant du fonctionnement du service public de la justice.

La reconnaissance de la compétence de l'autorité judiciaire aurait eu le mérite de qualifier clairement de judiciaires les décisions du juge de l'application des peines. De plus, les principes de la responsabilité de la puissance publique pourraient été utilisés, y compris la notion de risque et de responsabilité sans faute. La Cour de cassation a déjà admis la responsabilité pour risque ou rupture de l'égalité devant les charges publiques à propos de la police judiciaire ${ }^{59}$.

Ce débat sur la désignation d'une juridiction compétente n'est pas uniquement doctrinal. Il a des incidences sur l'évolution future des pratiques pénitentiaires car il est important de montrer que la prise en charge du détenu forme un ensemble cohérent que l'on ne peut pas séparer en fonction de l'autorité à l'origine de la décision.

Lorsque le droit admet d'engager la responsabilité de la puissance publique pour un dommage causé par un individu " responsable » mais pris en charge par le service public pénitentiaire, il fait le choix de considérer que, au moins comme en l'espèce, la permission de sortir ne rompt pas le lien qui unit le détenu à l'administration. L'activité du juge de l'application des peines n'est pas complètement indépendante de celle de l'Administration pénitentiaire. En participant activement aux missions confiées depuis 1987 au service public pénitentiaire par le législateur, ce magistrat développe une formule originale d'individualisation de la peine.

Il est même possible de dire qu'il développe considérablement l'idée de l'existence d'un service public de la justice, dont les magistrats sont les premiers agents. Le cas des permissions de sortir ou de la sortie sous escorte est particulièrement significatif de cette relation particulière entre le détenu et le juge de l'application des peines. Pour ces mesures, le magistrat ne se comporte pas comme un juge. La loi fait de lui le relais du service public pénitentiaire. Elle encourage ainsi le développement de la mission de resocialisation et impose de définir juridiquement la notion de garde et de prise en charge de la population pénale.

Pour améliorer le droit en prison, il est envisageable d'unifier et de simplifier l'ensemble du contentieux pénitentiaire en élargissant le débat classique sur le Tribunal d'application des peines ${ }^{60}$. Le Conseil constitutionnel ${ }^{61}$ a

51. CE 13 décembre 200o, Garde des Sceaux c. Primau et Fosset, req. $n^{\circ} 187698$.

52. Loi $\mathrm{n}^{\circ} 2000-516$ du 15 juin 2000.

53. M. Herzog-Evans, art. précité, Les Petites Affiches, $\mathrm{n}^{\circ}$ 169-170.

54. Fractionnement et suspension des peines, placement à l'extérieur, semi-liberté et libération conditionnelle.

55. Après avoir reconnu le principe du risque pénitentiaire, le juge a été amené à en examiner les conséquences respectives pour la semi-liberté (TA Toulouse, 14 mars 1969, MAIF c. Garde des Sceaux, req. ${ }^{\circ}$ 6690), la permission de sortir (TA Dijon, 2 juin 1981, Nicolet, req. $\mathrm{n}^{\circ} 8265$, Gaz. Pal. 24 novembre 1981, p. 22) ou la libération conditionnelle (TA Grenoble, Magnien, req. ${ }^{\circ} 14181$ ).

56. Conclusions Celier, sous CE, 30 avril 1948, Veuve Bernard, JCP, 1948-II-4133.

57. Conclusions Gazier, sous CE Sect., 25 janvier 1952, Vacqué, citées par G. Morange, D., 1952, p. 580.

58. Cass. Civ. 23 novembre 1956, Trésor public c. Giry, Bull. II, 407 ; GAJA, $\mathrm{n}^{\circ} 85$.

59. C. Cass. 10 juin 1986, Consorts Pourcel c. Pinier et Agent judiciaire du Trésor, RFDA, 1987, p. 92, note Buisson.

60. Un premier projet date de 1983 , il se fondait sur les différentes expériences étrangères: Projet de loi $\mathrm{n}^{\circ} 1723$ modifiant le Code de procédure pénale, le Code pénal et relatif à la personnalisation et à l'application des peines ainsi qu'à la révision des condamnations pénales, publiée au $J O$, annexe au procèsverbal de la séance du 3 octobre 1983.

61. Décision 89-261 DC du 28 juillet 1989, Loi relative aux conditions de séjour et d'entrée des étrangers en France, Rec. CC p. 81. 
explicitement prévu ce genre de situation : «Considérant cependant que, dans la mise en œuvre de ce principe, lorsque l'application d'une législation ou d'une réglementation spécifique pourrait engendrer des contestations contentieuses diverses qui se répartiraient, selon les règles habituelles de compétence, entre la juridiction administrative et judiciaire, il est loisible au législateur, dans l'intérêt d'une bonne administration de la justice, d'unifier les règles de compétences juridictionnelles au sens de l'ordre juridictionnel principalement intéressé. » La création d'une juridiction spécialisée capable de traiter de l'ensemble des questions pénitentiaires paraît donc être une solution possible pour peu que le législateur intervienne. L'unité du contentieux limiterait sensiblement les risques de disparités d'interprétation des grands principes pénitentiaires et permettrait de trouver un juste équilibre entre le respect de la légalité et la protection des libertés de chacun. 
